\title{
The Three-dimensional Endodontic Approach: A Novel Strategy to Deal with the Root Canal System
}

\author{
Gianluca Gambarini ${ }^{1}$, Gabriele Miccoli ${ }^{2}$, Rodolfo Reda ${ }^{3}$, Alessandro Mazzoni ${ }^{4}$, Luca Testarelli ${ }^{5}$
}

World Journal of Dentistry (2020): 10.5005/jp-journals-10015-1700

The introduction of cone beam computed tomography (CBCT) for endodontic diagnosis and treatment planning is probably one of the most significant innovations of the last years. When used appropriately, CBCT could provide useful and more detailed information that helps in the approach of complex cases, to simplify differential diagnosis, and consequently in the selection of the proper therapy. However, it is important that each case should be considered individually to ensure that the benefits provided are more than the risks of additional radiations. ${ }^{1}$ In the latest position statement edited in 2019 by the European Society of Endodontology on the use of $C B C T$ in endodontics, a wider range of scenarios where a scan may be useful is listed to improve the performance and facilitate the endodontic treatment. A two-dimensional (2D) preoperative periapical radiograph was considered for decades the main tool for diagnosis and treatment planning of endodontic issues. Unfortunately, a single periapical radiograph often offers poor visualization of the real anatomy and of all the pathologies affecting the tooth; this is mainly due to anatomical noise from superimposed structures, including bone and dentin, maxillary sinus, zygomatic arch, and inferior dental nerve; periapical lesions are also more difficult to diagnose in $2 \mathrm{D}$ periapical radiographs, since a dense cortical plate may hide them or their shape can be missed in a hypodense bone. ${ }^{2}$ In the past, identifying the number and the location of the canals' orifices has been considered a major clinical problem in endodontics because of many failures related to missed canals, but the use of preoperative $\mathrm{CBCT}$ has shown a significant improvement in reducing this risk; a three-dimensional (3D) scan can easily show the number of canals, the location of orifices, and the presence of confluences or diverging canals, not always detectable with the microscope. $^{3}$

Root canal anatomy and canal trajectories are very difficult to determine in a preoperative $2 \mathrm{D}$ periaphical radiograph: the $3 \mathrm{D}$ anatomy is compressed into a 2D image, which will result in the superimposition of the roots and canals: this could prevent full appreciation of the real anatomy. A CBCT scan, on the contrary, could efficiently provide this information before using any hand or rotary nickel-titanium instruments, also allowing a better assessment of canal complexities. ${ }^{4}$ Recently, novel software for analyzing endodontic parameters on CBCT images and reconstructing a $3 \mathrm{D}$-rendered image has been developed to allow a more interactive and user-friendly interface to better identify and visualize root canal trajectories, access cavity, the best straightline insertion of the instruments, and the initial determination of working length. ${ }^{5}$ Such information allows a better understanding of hidden curvatures and interference that could easily determine iatrogenic errors while instrumenting canals. ${ }^{6,7}$ Therefore, the use of preoperative $\mathrm{CBCT}$ scan to better understand endodontic anatomy
${ }^{1-5}$ Department of Oral and Maxillo-Facial Sciences, Sapienza University of Rome, Italy

Corresponding Author: Luca Testarelli, Department of Oral and Maxillo-Facial Sciences, Sapienza University of Rome, Italy, Phone: +39 3381504 134, e-mail: luca.testarelli@uniroma1.it

How to cite this article: Gambarini G, Miccoli G, Reda R, et al. The Three-dimensional Endodontic Approach: A Novel Strategy to Deal with the Root Canal System. World J Dent 2020;11(1):1-2.

Source of support: Nil

Conflict of interest: None

could represent a significant improvement aiming to provide a safer and more efficient root canal therapy. Recent researches started to investigate the possibility of using 3D images to perform guided root canal treatments and apicoectomies: this could represent the most promising novelty in the field. ${ }^{8-10}$ Since the root canal preparation is probably the key factor in the endodontic therapy, improving its quality by reducing the risk of iatrogenic errors during shaping and cleaning procedures will positively affect the final outcome.

\section{References}

1. Gambarini G, Piasecki L, Miccoli G, et al. Cone-beam computed tomography in the assessment of periapical lesions in endodontically treated teeth. Eur J Dent 2018;12(1):136-143. DOI: 10.4103/ejd. ejd_320_17.

2. Gambarini G, Miccoli G, Gaimari G, et al. Detection of bone defects using CBCT exam in an Italian population. Int J Dent 2017;2017:7523848. DOI: 10.1155/2017/7523848.

3. Connert T, Zehnder MS, Amato M, et al. Microguided endodontics: a method to achieve minimally invasive access cavity preparation and root canal location in mandibular incisors using a novel computerguided technique. Int Endod J 2018;51(2):247-255. DOI: 10.1111/ iej.12809.

4. Gambarini G, Plotino G, Grande NM, et al. Differential diagnosis of endodontic-related inferior alveolar nerve paraesthesia with cone beam computed tomography: a case report. Int Endod J 2011;44(2):176-181. DOI: 10.1111/j.1365-2591.2010.01816.x.

5. Segato AVK, Piasecki L, Felipe Iparraguirre Nuñovero M, et al. The Accuracy of a new cone-beam computed tomographic software in the preoperative working length determination ex vivo. J Endod 2018;44(6):1024-1029. DOI: 10.1016/j.joen.2018.02.027.

6. Gambarini G, Piasecki L, Miccoli G, et al. Classification and cyclic fatigue evaluation of new kinematics for endodontic instruments. Aust Endod J 2019;45(2):154-162. DOI: 10.1111/aej.12294.

7. Gambarini G, Miccoli G, Seracchiani M, et al. Fatigue resistance of new and used nickel-titanium rotary instruments: a comparative study. Clin Ter 2018;169(3):e96-e101. DOI: 10.7417/T.2018.2061.

(O) The Author(s). 2020 Open Access This article is distributed under the terms of the Creative Commons Attribution 4.0 International License (https://creativecommons. org/licenses/by-nc/4.0/), which permits unrestricted use, distribution, and non-commercial reproduction in any medium, provided you give appropriate credit to the original author(s) and the source, provide a link to the Creative Commons license, and indicate if changes were made. The Creative Commons Public Domain Dedication waiver (http://creativecommons.org/publicdomain/zero/1.0/) applies to the data made available in this article, unless otherwise stated. 
8. Zehnder MS, Connert T, Weiger R, et al. Guided endodontics: accuracy of a novel method for guided access cavity preparation and root canal location. Int Endod J 2016;49(10):966-972. DOI: 10.1111/iej.12544.

9. Gambarini G, Galli M, Stefanelli LV, et al. Endodontic microsurgery using dynamic navigation system: a case report.
J Endod 2019;45(11):1397-1402. DOI: 10.1016/j.joen.2019. 07.010 .

10. Moreno-Rabié C, Torres A, Lambrechts $P$, et al. Clinical applications, accuracy and limitations of guided endodontics: a systematic review. Int Endod J 2020;53(2):214-231. DOI: 10.1111/iej.13216. 